\title{
The Effect of Negative Poisson's Ratio Polyurethane Scaffolds for Articular Cartilage Tissue Engineering Applications
}

\author{
Yeong Jun Park and Jeong Koo Kim \\ Department of Biomedical Engineering, Graduate School, Inje University, Gimhae 621-749, Republic of Korea \\ Correspondence should be addressed to Jeong Koo Kim; jkkim@inje.ac.kr
}

Received 16 May 2013; Accepted 21 August 2013

Academic Editor: Hamdy Doweidar

Copyright ( 2013 Y. J. Park and J. K. Kim. This is an open access article distributed under the Creative Commons Attribution License, which permits unrestricted use, distribution, and reproduction in any medium, provided the original work is properly cited.

\begin{abstract}
An auxetic polyurethane (PU) scaffold was prepared to investigate chondrocyte proliferation under compressive stimulation for cartilage regeneration. To give a negative Poisson's ratio to the PU scaffold, volumetric compression with a $3: 1$ ratio was applied during heat treatment. For the control PU scaffold, the Poisson's ratio was $0.9 \pm 0.25$ with elongation at $20 \%$ of the strain range. Poisson's ratio for experimental specimens was approximately $-0.4 \pm 0.12$ under the same conditions. In cell proliferation tests, cells were cultivated within the prepared scaffold under compression with a $20 \%$ strain range. With a $20 \%$ strain range elongation, the compressive load was approximately $0.3 \mathrm{~N}$. The experimental group showed a 1.3 times higher cellular proliferation rate than that of the control group after 3 days in culture. At day 5 of culture, however, the rate of proliferation of the control group increased so that there was no significant difference between groups. However, collagen content (produced by the cells) in the cell-proliferated medium was 1.5 times higher in the experimental group after 5 days in culture. This may have been due to the effectiveness of the auxetic structure of the scaffold. An isotropic compressive load was transmitted to the cells due to the negative Poisson ratio of the scaffold.
\end{abstract}

\section{Introduction}

Bone and cartilage generation by autogenous cell/tissue transplantation is one of the most promising techniques in orthopedic surgery and biomedical engineering [1]. Due to the inability of avascular and aneural tissues to self-heal, even minor cartilage defects can result in mechanical joint instability and progressive damage, and cartilage damage is notoriously difficult to treat and cure [2]. Autologous cell-based tissue engineering using three-dimensional (3D) porous scaffolds has provided an option for the repair of full thickness defects in adult cartilage tissue [3]. The scaffold or $3 \mathrm{D}$ construct provides the necessary support for cells to proliferate and maintain their differentiated function, and its architecture defines the ultimate shape of the new bone and cartilage [4].

All these natural and artificial materials for 3D scaffolds have a convex cell shape and exhibit a positive Poisson's ratio, which is defined as the negative of the lateral strain divided by the longitudinal strain when a load is applied in the longitudinal direction [5]. On the other hand, materials or structures that contract in the transverse direction under uniaxial compression or expand laterally when stretched are said to have negative Poisson's ratios $[6,7]$. This phenomenon is the opposite of the behavior of a positive Poisson's ratio due to the effect of the material structure. For negative Poisson's ratio material, the structures are commonly known as auxetic structures, which are similar to a reentrant honeycomb structure [8]. This reentrant shape is fabricated by transforming a conventional honeycomb shape into the concave structure that finally produces the auxetic structure. As shown in Figure 1, the reentrant structure unfolds under tension and gives rise to a negative Poisson's ratio. Usually, an allowable range of Poisson's ratio is -1.0 to 0.5 based on the thermodynamic consideration of strain energy in the theory of elasticity [9]. In the last two decades, the behavior of the negative Poisson's ratio was predicted, discovered, or deliberately introduced in various materials, such as foam and microstructure polymers $[10,11]$. This sort of material is expected to have interesting mechanical properties, such 


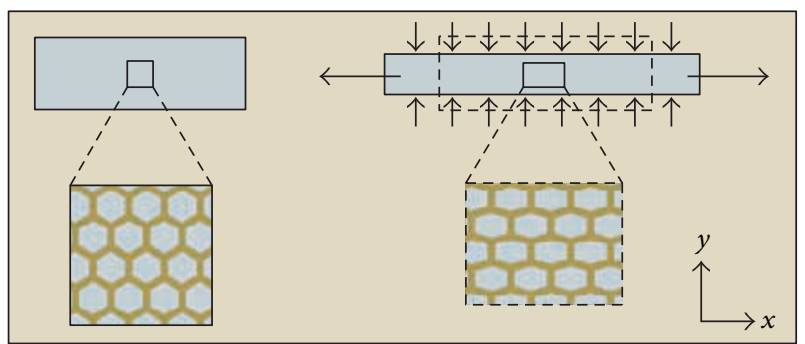

(a)

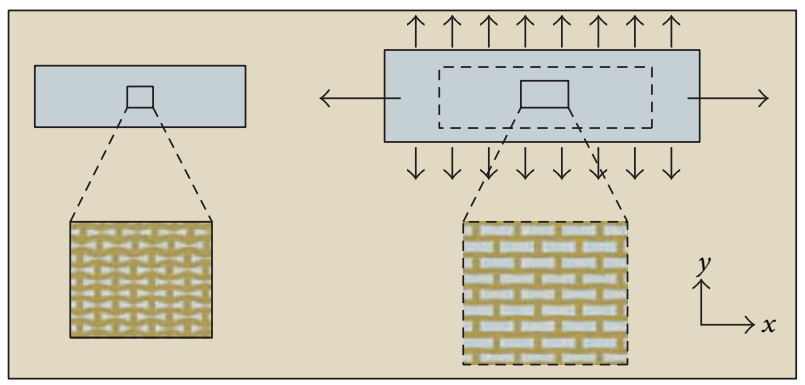

(b)

FIGURE 1: Behaviour of positive Poisson's ratio and negative Poisson's ratio when subjected to tensile loading. The pores have a rounder shape (a), while in (b), the pore space is larger, giving rise to a negative Poisson's ratio [11].

as high energy absorption, fracture toughness, indentation resistance, and enhanced shear moduli, which may be useful in some applications [6].

Auxetic materials are known to exhibit various enhanced physical characteristics over their conventional counterparts [10]. They show increased indentation resistance and improved acoustic damping properties. These enhanced characteristics make negative Poisson's ratio materials perform better in many practical applications; however, although these enhanced properties have been studied in industrial and academic fields, there have been few studies in tissue engineering. Fozdar et al. reported that there are possibilities to fabricate tunable negative Poisson's ratio scaffold by digital micromirror device projection printing for tissue engineering applications [12].

In cartilage tissue engineering, a focus has been on the relationship between chondrocyte response and physical properties of the scaffold (i.e., stiffness, hydrophilicity). However, knowledge is lacking about chondrocyte behavior with mechanical stimulation in a negative Poisson's ratio scaffold. Mechanical stimulation associated with normal body functions is crucial in properly reforming articular cartilage with tissue engineering [2].

In this study, we form an auxetic structural polyurethane (PU) scaffold for cartilage regeneration and investigate chondrocyte proliferation effectiveness within the auxetic scaffold under mechanical (compression) stimulation.

\section{Materials and Methods}

2.1. Specimen (Scaffold) Preparation. PU foam was purchased from Kumsung Sponge Co. (Seoul, Republic of Korea).

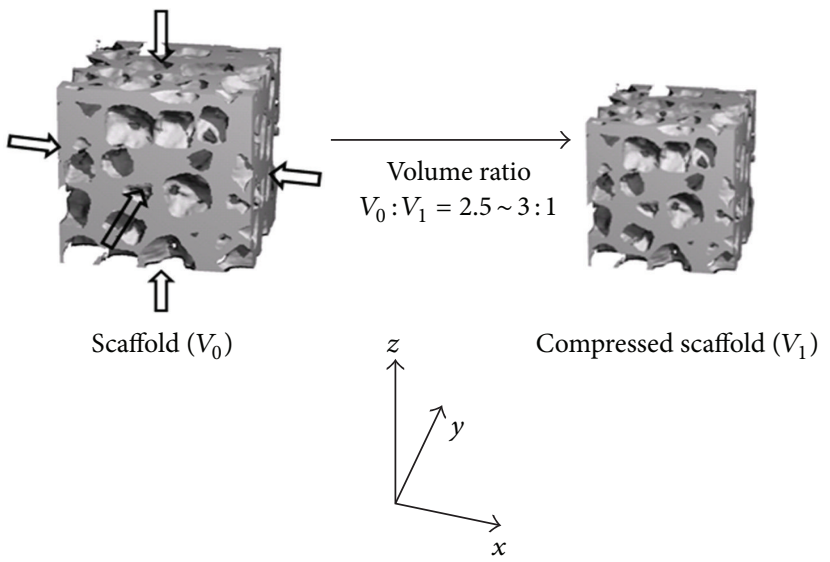

Figure 2: Schematic diagram of the preparation of an auxetic scaffold (compressive volume ratio $=2.5 \sim 3.0$ ).

PU foams were treated with $70 \%$ ethanol for $30 \mathrm{~min}$ for sterilization.

Control specimens were prepared with 60 ppi PU foam. Pore size and shape of experimental specimens were controlled by heating and compression. A metal mold with dimensions of $5 \mathrm{~cm} \times 5 \mathrm{~cm} \times 7.5 \mathrm{~cm}$ was used to prepare specimens. To materialize the auxetic structure, the desired volumetric ratio was estimated as 3.0 based on a predetermined calculation. A schematic diagram of the process is presented in Figure 2. The initial sample size of the PU sample was cut to approximately $8 \mathrm{~cm} \times 8 \mathrm{~cm} \times 9 \mathrm{~cm}$, giving an initial volume to final volume ratio of $1: 3$ after applying compression. The PU foam was then placed carefully into the mold, using a tongue depressor to prevent wrinkles. The foam was pulled and adjusted at each end to ensure that it fit the mold exactly. The metallic mold was then placed in the center of a furnace (preheated to $150^{\circ} \mathrm{C}$ ) for $30 \mathrm{~min}$ at $200^{\circ} \mathrm{C}$ under triaxial compression in order to produce a reentrant structure of the PU foam. Then, the mold was cooled slowly to room temperature. The mold containing the PU foam was removed from the furnace, and the heat-treated PU foam was removed from the mold and cut into small cylindrical shapes.

2.2. Estimation of Poisson's Ratio. Poisson's ratio was estimated by image processing $[13,14]$. A digital microscope (BX51; Olympus Corporation, Tokyo, Japan) was used to measure Poisson's ratio of the specimens. Compressive loading was applied to the specimens with a material testing machine (LRX-PLUS; Lloyd Instruments, West Sussex, UK). The experimental setup with the microscope for capturing the specimen while applying a load to measure the displacement of the specimen is shown in Figure 3. The relative positions of pointed marks were used to estimate the strain and Poisson's ratio. Images were processed with Photoshop 7.0. For each specimen, five measurements were taken from a 0.05 to 0.25 strain range, as shown in Figure 4 . In-built tools of the digital microscope were used to measure the $x$-axis and $y$ axis displacements, which were then calculated using (1) to determine Poisson's ratio. 


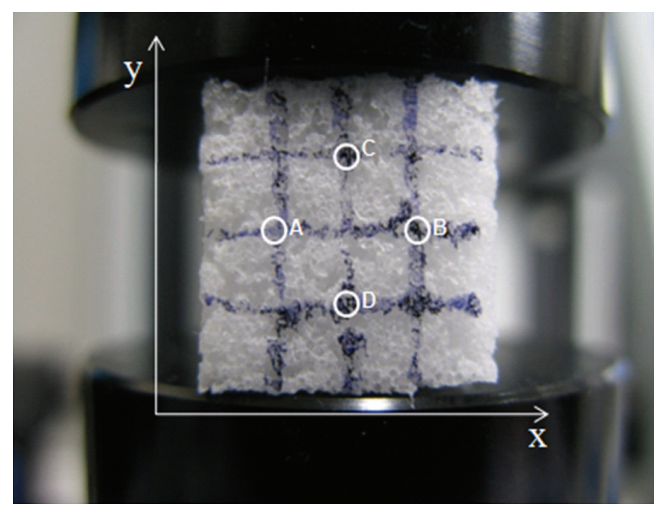

FIGURE 3: Experimental setup with microscope for capturing the specimen while applying a load to measure the displacement of the specimen with the points (A to D).

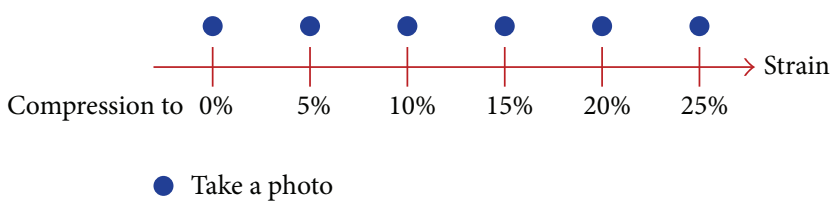

FIGURE 4: Strain intervals while applying load for taking photos to measure displacement of the prepared specimens.

In order to investigate pore shapes and distribution, a cross section of the scaffold was observed by Scanning Electron Microscope (S-4300DSE, HITACHI, Tokyo, Japan). We have

$$
\begin{gathered}
\varepsilon_{x}=\frac{|A-B|-\left|A_{0}-B_{0}\right|}{\left|A_{0}-B_{0}\right|}, \\
\varepsilon_{y}=\frac{|C-D|-\left|C_{0}-D_{0}\right|}{\left|C_{0}-D_{0}\right|}, \\
v=-\frac{\varepsilon_{x}}{\varepsilon_{y}},
\end{gathered}
$$

where $\varepsilon_{x}=$ strain of $x$-axis, $\varepsilon_{y}=$ strain of $y$-axis, $\nu=$ material Poisson's ratio, $A_{0}$ to $D_{0}=$ initial point $($ load $=0)$, and $A$ to $D$ $=$ moved point under load.

2.3. Chondrocyte Isolation and Culture. Porcine cartilage was harvested from the lateral and medial condyle of 6-monthold pig hinge legs. The obtained cartilage was chopped into small pieces with sterilized scissors. The cartilage was digested for $4 \mathrm{~h}$ in Dulbecco's Modified Eagle Medium (DMEM, Gibco) containing $2 \mathrm{mg} / \mathrm{mL}$ collagenase and filtered through a nylon sieve cell strainer (Falcon, New Jersey, USA) having an $80 \mu \mathrm{m}$ pore size. The cells were rinsed with phosphate buffered saline (PBS) solution (Lonza, Walkersville, USA) and then centrifuged at $1500 \mathrm{rpm}$ for $5 \mathrm{~min}$. The centrifuged cell pellet was washed with PBS, and the number of cells was determined by using a hemocytometer. Chondrocytes were then cultured in cell culture flasks with DMEM supplemented with 10\% fetal bovine serum (Gibco) and $1 \%$ antibiotics (Gibco) in a humidified incubator with 5\% $\mathrm{CO}_{2}$ at $37^{\circ} \mathrm{C}$. The media were exchanged every 3 days.

2.4. Cell Proliferation Estimation. For sterilization of the specimens in order to culture the prepared cells, all specimens were incubated with $70 \%$ ethanol for $1 \mathrm{~h}$, followed by washing three times with PBS solution. For cell seeding, second to third passage chondrocytes were used. An initial cell density of $2.0 \times 10^{5}$ (cells/specimen) was seeded in a humidified incubator containing $5 \% \mathrm{CO}_{2}$ at $37^{\circ} \mathrm{C}$. Cells were cultured in the prepared scaffold with static compression (compression with $20 \%$ strain). The exerted static load from $20 \%$ strain elongation of the prepared scaffold was about $0.3 \mathrm{~N}$. The cellular proliferation rate was measured by using the Cell Counting Kit (CCK-8; Dojindo Molecular Technologies, Inc., Maryland, USA) and the Sircol collagen assay kit (Biocolor, Antrim, UK). Experiments were observed on days 1, 3, and 5. The absorbances of the CCK- 8 solution (DMEM:CCK solution $=10: 1)$ and Sircol collagen assay solution were measured by using a Fluorescence Multi-Detection Reader (Synergy HT, BIO-TEK Instruments, Inc., Vermont, USA) at $450 \mathrm{~nm}$ and $550 \mathrm{~nm}$, respectively.

2.5. Statistical Analysis. Data were expressed as mean \pm standard error for all comparisons. A $t$-test was used to evaluate differences between groups $(P<0.05)$.

\section{Results and Discussion}

A PU scaffold with a negative Poisson's ratio was formed by creating a reentrant cell structure inside the foam. The original microstructure of the PU foam is shown in Figure 5(a); the pores are well distributed and uniform in shape and size. For formation of negative Poisson's ratio scaffolds, a compressive load was applied with thermal treatment; this resulted in the transformation of the microstructure from a convex cell shape to a deformed cell shape as shown in Figure 5(b). The cell shapes were deformed and generated new bonding and debonding between the cells [15]. As a result, Poisson's ratio of the control group was estimated as $0.9 \pm 0.25$ on average with $20 \%$ strain elongation via compressive loading. In contrast, the experimental group had a Poisson's ratio of approximately $-0.4 \pm 0.12$ with $20 \%$ strain elongation via compressive loading (Figure 6).

In the cellular proliferation test, the experimental group had a 1.3 times higher cellular proliferation rate compared to the control group 3 days after cell seeding. At day 5 in culture, the experimental group had a 1.2 times higher cellular proliferation rate, but there was no significant difference between groups, as shown in Figure 7. However, results of the Sircol collagen assay showed that the amount of collagen produced by the cells after 3 and 5 days in culture was about 1.5 times higher in the experimental group $(P<0.05)$, as shown in Figure 8. This is an important finding because collagen production is essential for proliferated cells to construct extracellular matrix in tissue engineering. The results suggest that isotropic compressive stimulation occurred due to the auxetic structure of the scaffold and isotropic mechanical stimulation was effectively transmitted to the cells through 


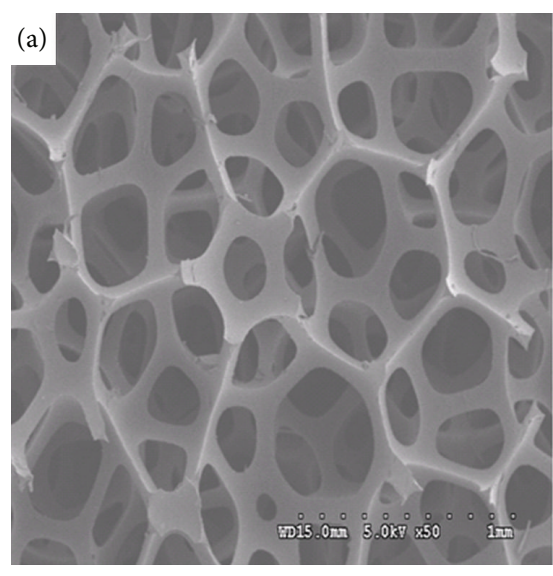

(a)

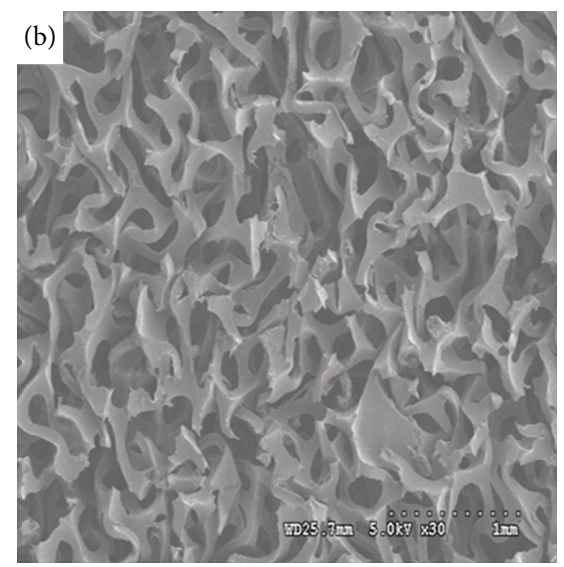

(b)

FIGURE 5: Microstructures of polyurethane scaffolds: (a) a control specimen and (b) an experimental specimen.

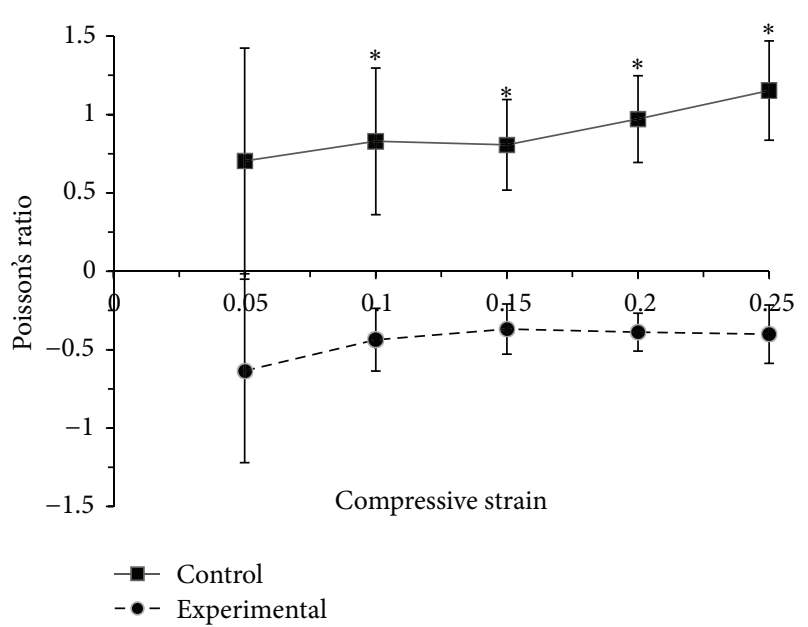

FIGURE 6: Poisson's ratio variation of prepared polyurethane scaffolds with strain ranges with compressive loading $\left({ }^{*} P<0.05, n=5\right)$.

the scaffold. Isotropic compressive stimulation was favorable for chondrocyte proliferation and collagen production. The results indicate that isotropic compressive stimulation could be more effective on collagen production during chondrocyte proliferation, which is a good indicator for the construction of extracellular matrix as well as cartilage tissue, and that chondrocytes preferentially proliferate on a negative Poisson's ratio PU scaffold rather than a conventional scaffold because of isotropic mechanical stimulation.

The cellular proliferation decrease after 5 days in culture could be affected by viscoelastic properties of the scaffold. The applied load to the scaffold would be transmitted sufficiently to the cells during the initial 3 days; however, on day 5 , the compressive load should be decreased due to stress relaxation of the PU scaffold. Hence, it is inferred that the increase in cellular proliferation decreased at day 5 compared with the initial 3-day cultivation period.

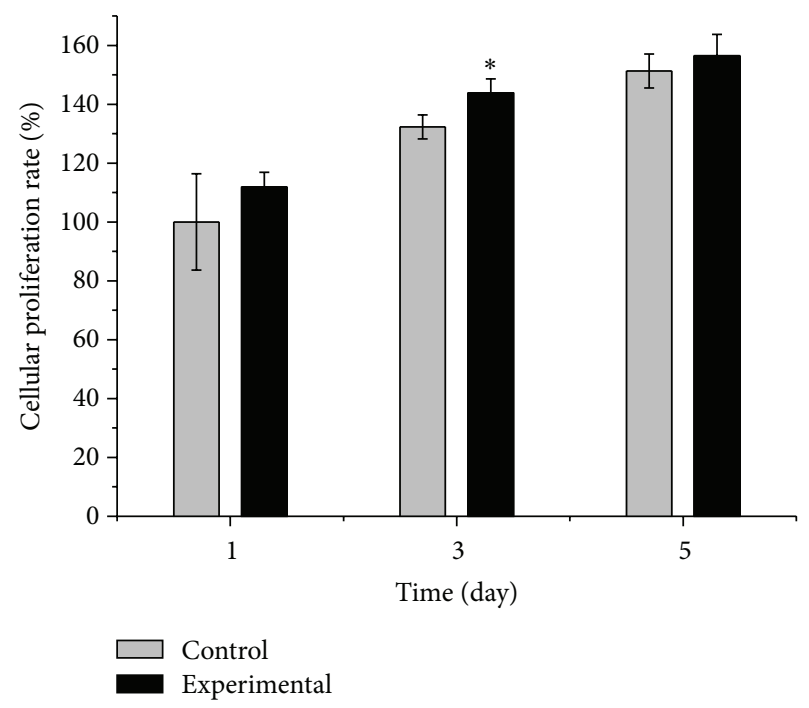

FIGURE 7: Chondrocyte proliferation rate on polyurethane scaffolds. Values were normalized $\left(n=5,{ }^{*} P<0.05\right)$.

\section{Conclusions}

It is well known that physical and/or chemical stimulation is effective for cellular proliferation during cell cultivation. We used PU foam to investigate the formation of an auxetic structural scaffold and the effectiveness of cell proliferation in the scaffold with mechanical stimulation. With compressive load stimulation, the auxetic PU scaffold successfully transferred the compressive load isotropically to the cells. Hence, the auxetic scaffold could be effective for chondrocyte proliferation with compressive load stimulation. From this study, we confirmed that mechanical stimulation is effective for chondrocytes proliferation and that isotropic stimulation could be more effective for collagen production from the cells.

Biodegradable polymeric scaffolds are widely used in tissue engineering, but there have been few studies related to auxetic biodegradable scaffolds. Hence, we suggest that 


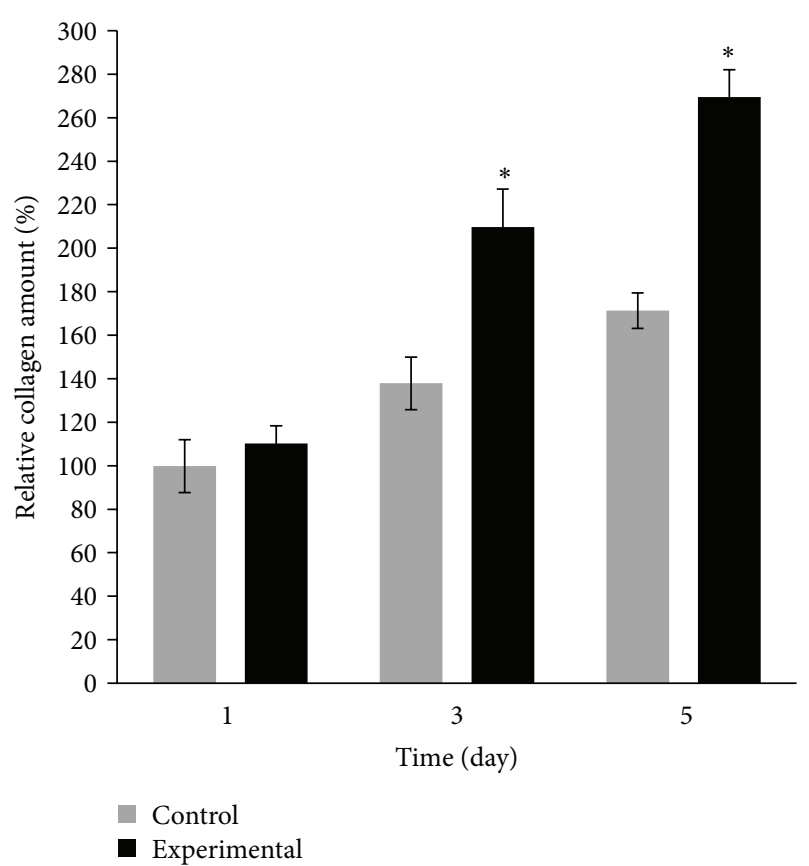

FIGURE 8: Relative amount of collagen within the PU scaffold during cell cultivation. Values were normalized $\left(n=5,{ }^{*} P<0.05\right)$.

biodegradable polymeric scaffolds should be considered for forming an auxetic structure as well as for isotropic mechanical stimulation in cartilage tissue engineering.

\section{Acknowledgment}

This work was supported by a 2013 Inje University research grant.

\section{References}

[1] C. W. Patrick Jr., A. G. Mikos, and L. V. McIntire, "Prospectus of tissue engineering," in Frontiers in Tissue Engineering, pp. 3-14, Elsevier Science, New York, NY, USA, 1998.

[2] Y. M. Jung, M. S. Park, J. W. Lee et al., "Cartilage regeneration with highly-elastic three-dimensional scaffolds prepared from biodegradable poly(l-lactide-co- $\varepsilon$-caprolactone)," Biomaterials, vol. 29, no. 35, pp. 4630-4636, 2008.

[3] Y. Wang, D. J. Blasioli, H. J. Kim, H. S. Kim, and D. L. Kaplan, "Cartilage tissue engineering with silk scaffolds and human articular chondrocytes," Biomaterials, vol. 27, no. 25, pp. 44344442, 2006.

[4] D. W. Hutmacher, "Scaffolds in tissue engineering bone and cartilage," Biomaterials, vol. 21, no. 24, pp. 2529-2543, 2000.

[5] J. B. Choi and R. S. Lakes, "Analysis of elastic modulus of conventional foams and of re-entrant foam materials with a negative Poisson's ratio," International Journal of Mechanical Sciences, vol. 37, no. 1, pp. 51-59, 1995.

[6] H. Wan, H. Ohtaki, S. Kotosaka, and G. Hu, "A study of negative Poisson's ratios in auxetic honeycombs based on a large deflection model," European Journal of Mechanics, A/Solids, vol. 23, no. 1, pp. 95-106, 2004.
[7] B. Brandel and R. S. Lakes, "Negative Poisson's ratio polyethylene foams," Journal of Materials Science, vol. 36, no. 24, pp. 5885-5893, 2001.

[8] D. Prall and R. S. Lakes, "Properties of a chiral honeycomb with a Poisson's ratio of -1," International Journal of Mechanical Sciences, vol. 39, no. 3, pp. 305-314, 1997.

[9] H. Ohtaki, G. Hu, Y. Nagasaka, and S. Kotosaka, "Analysis of negative Poisson's ratios of re-entrant honeycombs," JSME International Journal A, vol. 47, no. 2, pp. 113-121, 2004.

[10] J. N. Grima, R. Gatt, N. Ravirala, A. Alderson, and K. E. Evans, "Negative Poisson's ratios in cellular foam materials," Materials Science and Engineering A, vol. 423, no. 1-2, pp. 214-218, 2006.

[11] W. Yang, Z. M. Li, W. Shi, B. H. Xie, and M. B. Yang, "Review on auxetic materials," Journal of Materials Science, vol. 39, no. 10, pp. 3269-3279, 2004.

[12] D. Y. Fozdar, P. Soman, J. W. Lee, L.-H. Han, and S. Chen, "Three-dimensional polymer constructs exhibiting a tunable negative Poisson's ratio," Advanced Functional Materials, vol. 21, no. 14, pp. 2712-2720, 2011.

[13] J. B. Choi and R. S. Lakes, "Nonlinear properties of polymer cellular materials with a negative Poisson's ratio," Journal of Materials Science, vol. 27, no. 17, pp. 4678-4684, 1992.

[14] R. D. Widdle Jr., A. K. Bajaj, and P. Davies, "Measurement of the Poisson's ratio of flexible polyurethane foam and its influence on a uniaxial compression model," International Journal of Engineering Science, vol. 46, no. 1, pp. 31-49, 2008.

[15] A. Butenhoff, R. S. Lakes, and Y. C. Wang, "Influence of cell size on re-entrant transformation of negative Poisson's ratio reticulated polyurethane foams," Cellular Polymers, vol. 20, no. 4-6, pp. 373-385, 2001. 

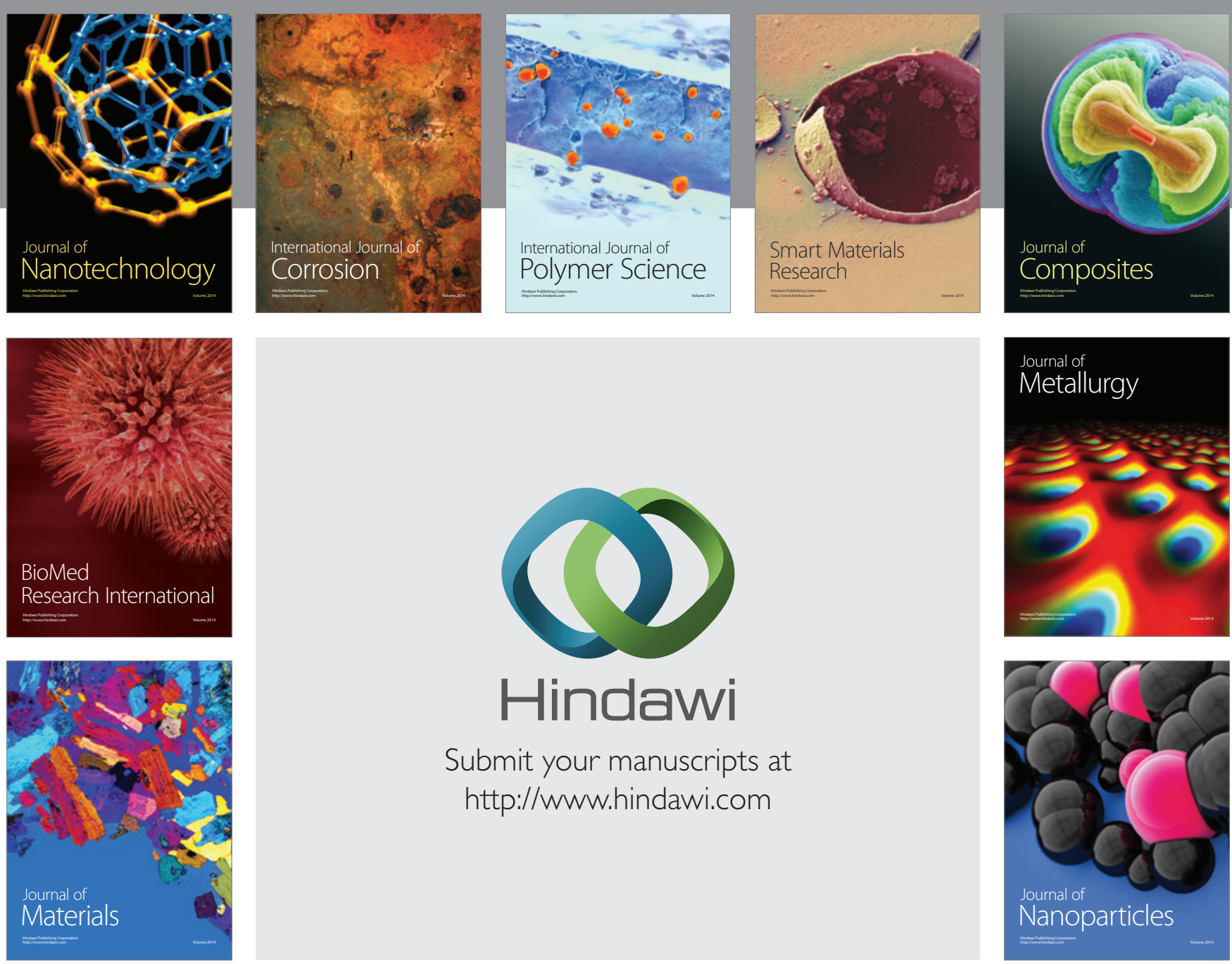

Submit your manuscripts at http://www.hindawi.com
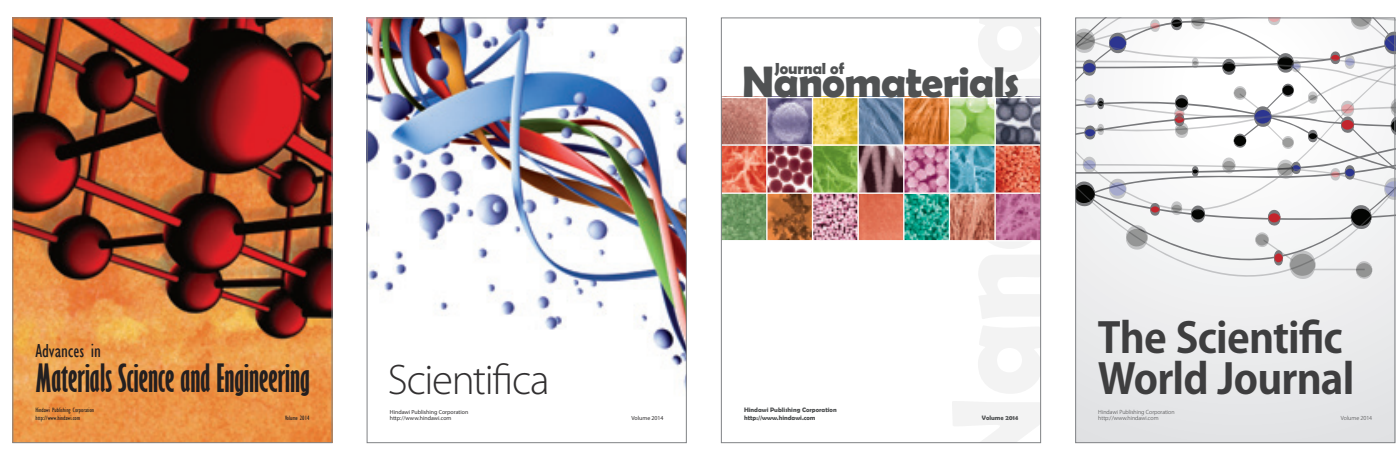

\section{The Scientific World Journal}
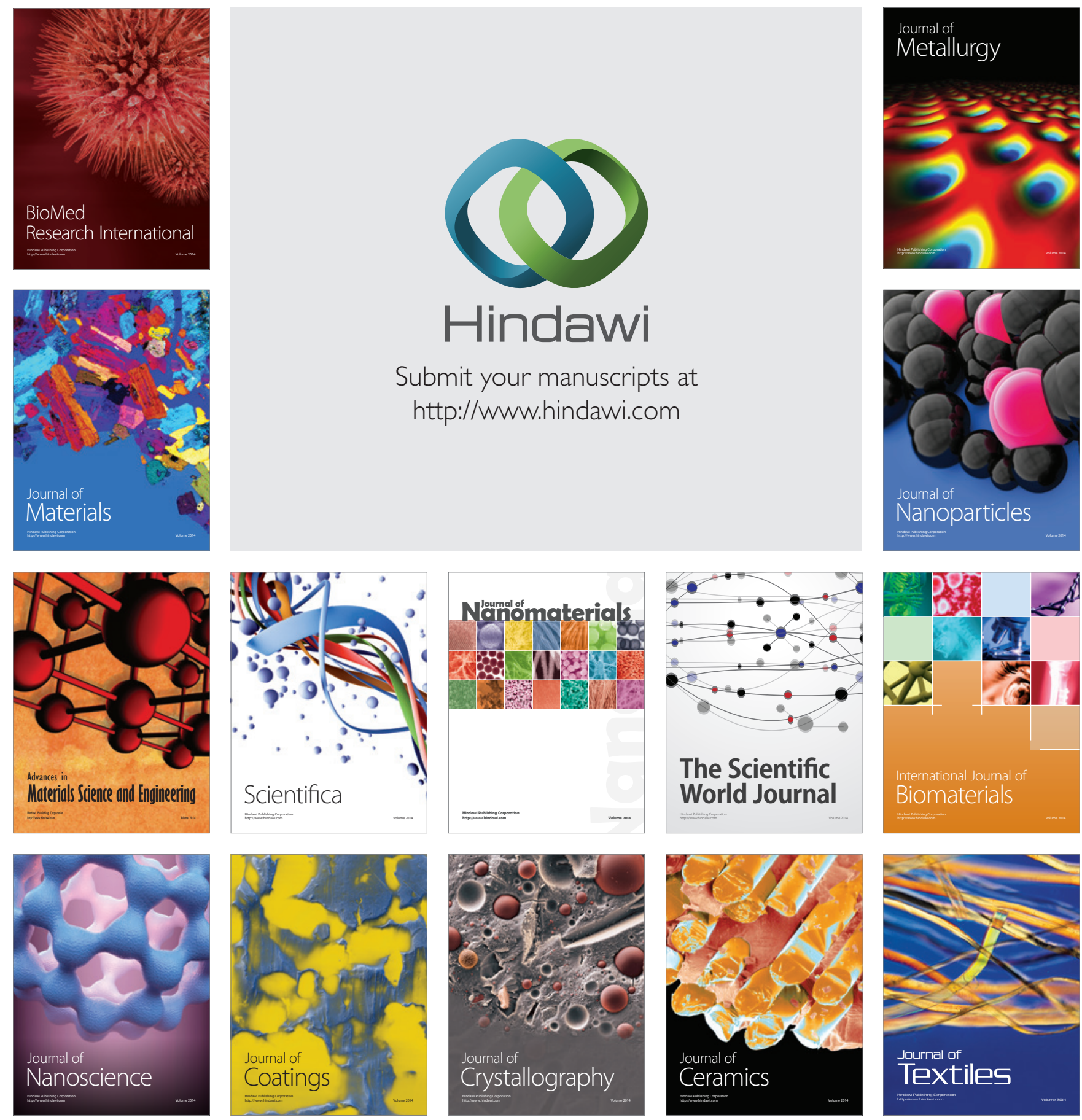